\title{
Verbal to visual code switching improves working memory in older adults: an fMRI study
}

\author{
Mariko Osaka', Yuki Otsuka ${ }^{2}$ and Naoyuki Osaka ${ }^{2 *}$ \\ Department of Psychology, Graduate School of Human Sciences, Osaka University, Osaka, Japan \\ ${ }^{2}$ Department of Psychology, Graduate School of Letters, Kyoto University, Kyoto, Japan
}

\author{
Edited by: \\ Joel Pearson, The University of \\ New South Wales, Australia \\ Reviewed by: \\ Claude Alain, Rotman Research \\ Institute, Canada \\ Axel Kohler, University of Münster, \\ Germany \\ ${ }^{*}$ Correspondence: \\ Naoyuki Osaka, Department of \\ Psychology, Graduate School of \\ Letters, Kyoto University, \\ Yoshida-Honmachi, Sakyo-ku, \\ Kyoto 606-8501, Japan. \\ e-mail:nosaka@bun.kyoto-u.ac.jp
}

\begin{abstract}
The effect of verbal to visual code switching training on working memory performance was investigated in individuals aged 63 and older. During verbal working memory task performance, the training group $(n=25)$ was introduced to a verbal to visual code switching strategy while the control group $(n=25)$ was not exposed to such a strategy. Working memory recognition accuracy was enhanced only in the training group. To explore the neural substrates underlying these strategy effects, fMRI was used to measure brain activity in both groups during working memory task performance before and after an attention training period. In a comparison between pre- and post-training sessions, results showed increased activation in the anterior cingulate cortex (ACC). Relative to the control group, the post-training group exhibited increased activation in the left and right inferior parietal lobules (IPLs) and right superior parietal lobule (SPL). These findings suggest that use of a verbal to visual code switching strategy may assist older individuals in the maintenance of information in working memory.
\end{abstract}

Keywords: working memory, older adults, fMRI, strategy training, attention switching

\section{INTRODUCTION}

Working memory serves simultaneous information, storage, and processing functions (Baddeley, 1986). According to the multicomponent model proposed by Baddeley (Baddeley, 1986, 2003; Baddeley and Logie, 1999), working memory is composed of two storage buffers: the phonological loop for storing verbal information and the visuo-spatial sketchpad for the storage of visual information. Because working memory resources are limited, an executive system assigns and coordinates these limited resources for storing and manipulating information (Daneman and Carpenter, 1980; Baddeley and Logie, 1999; Engle et al., 1999).

Working memory impairments are commonly found in older adults. In particular, older individuals often have difficulty performing dual tasks that require both storage and processing (Salthouse, 1996; West, 1996; Craik and Salthouse, 2000). It has been proposed that vulnerability of the frontal lobes accounts for the age-related impairments in memory functioning found in older adults (Raz et al., 1997; Raz, 2000; Madden et al., 2004). In accordance with this hypothesis, decreased prefrontal cortex (PFC) activation has been found in older adults through neuroimaging studies (Bunge et al., 2000; Cabeza, 2001). Age-related reductions in the ability to suspend non-task-related activity and engage areas for carrying out memory tasks have also been reported (Grady et al., 2006).

Several studies have examined whether training improves working memory in older adults (Nyberg et al., 2003; Erickson et al., 2007). Erickson et al. (2007) found that dual-task training induced performance improvements which were correlated with an increase in hemispheric asymmetry and a reduction in age differences in ventral and dorsal PFC activation. The investigators argued that these results provide evidence of brain plasticity in older individuals. Other studies indicated that training in the $n$-back task, which requires executive control of working memory, is associated with improvements in the working memory performance of older adults (Dahlin et al., 2008a; Li et al., 2008). Dahlin et al. (2008b) showed that training in updating information, which requires executive function in working memory, improved significantly in young and older adults relative to controls. Based on these reports, it is likely that the dual-task deficits typically observed in older individuals are caused not only by a decreased ability to maintain information in working memory, but also by deficits in the executive processing system.

The neural basis of the working memory system, particularly executive functioning, is thought to be located in the PFC; recent neuroimaging studies have suggested that the executive system is located in the dorsolateral PFC (DLPFC, BA9/46) and anterior cingulate cortex (ACC) (D'Esposito et al., 1995, 1999; Smith et al., 1996; Cohen et al., 1997; Owen et al., 1998; Smith and Jonides, 1999; Bunge et al., 2000; Osaka et al., 2003, 2004; Linden, 2007). The brain regions underlying working memory appear to drive different executive functions. The DLPFC supports the active maintenance of attention on task goals, whereas the ACC serves as an attentional coordinator, which enables individuals to inhibit inappropriate stimuli while monitoring task performance (MacDonald et al., 2000). ACC activation appears to increase when cognitive tasks require greater attentional demands (Petersen et al., 1988; Posner and Petersen, 1990).

Osaka et al. $(2003,2004)$ used the reading span test (RST) to compare PFC activation in high-performing normal young adults with those of low performers, and found that ACC and DLPFC activation was greater in high versus low performers. The RST is 
a commonly used dual task developed to measure working memory resources available for reading sentences and storing a target word associated with each sentence (Daneman and Carpenter, 1980; Just and Carpenter, 1992). Individual differences in working memory capacity are likely derived from recruited activity shared in the DLPFC and ACC (Osaka et al., 2003). In addition to these results for young adults, our previous research in older adults showed less ACC activation relative to younger individuals (Otsuka et al., 2006, 2008). Based on these findings, it is plausible to assume that working memory impairments in older adults are due to ineffective attentional coordination, as supported by a network including the ACC and DLPFC.

In the present study, we investigated the effect of attentional coordination training on dual-task performance in older adults. Strategic training is considered to be an effective method for increasing working memory performance (McNamara and Scott, 2001). For example, strategic training using visual imagery produces increased correct recall performance when older individuals perform a paired association task (Yesavage, 1983; Carretti et al., 2007). In our previous study (Osaka and Nishizaki, 2000) we found that high-RST performers tended to use a mental imagery strategy more often than low-RST performers, who appear to restrict themselves to verbal rehearsal. Based upon Baddeley's multi-component model of working memory (Baddeley, 2003), we introduced for the first time a unique training task that required participants to switch attention from verbal to visual images, (i.e., switching from phonological information to visuo-spatial information), with the intention that this approach would strengthen the executive control function of working memory in older adults. To our knowledge, there have been no published reports on the use of visual image formation as a training strategy to improve working memory in older adults. We hypothesized that attention switching training through the use of image formation would improve elder participants' working memory performance.

To investigate the neural substrates underlying such training effects, we measured fMRI activation while participants performed a working memory task (the RST) before and after the training period. We hypothesized that this training of attention switching from verbal to visual images activated brain regions related to attentional control. Moreover, it is predicted that an activation increase would be found in both the left and right hemispheres according to the engagement of phonological and visuo-spatial information processing simultaneously.

\section{MATERIALS AND METHODS PARTICIPANTS}

Fifty volunteers participated in the experiment. All participants reported that they had no history of neuropsychological or psychiatric disease and all were right-handed. Their educational levels ranged from 9 to 16 years $($ mean $=12.76, \mathrm{SD}=1.9$ ).

Participants performed the RST in a preliminary behavioral screening using a paper version. In the RST, participants were required to read aloud sets of one to five sentences presented one at a time and to remember a target word for each sentence. Each sentence condition included five trials, and all participants completed five total trials in the experiment. Span score was calculated as the highest level at which a participant could recall all words from the list in three of the five trials. Overall performance on the RST was assessed in terms of the total number of words recalled. RST scores of the participants ranged from 1.5 to 4.0.

Both the training and control groups were matched for age, educational level, and RST estimates in the screening test. Pretest, training, and post-test sessions all occurred on the same day. Twenty-five participants ( 17 males and eight females, age range $=$ 63-73 years, mean $=68.08, \mathrm{SD}=2.7$, educational level range $=$ $9-16$, mean $=12.0, \mathrm{SD}=2.3)$ participated in the strategy training (training group), whereas the other 25 (15 males and 10 females, age range $=63-75$ years, mean $=68.50, \mathrm{SD}=3.4$, educational level range $=9-16$, mean $=11.8, \mathrm{SD}=2.0$ ) did not receive training (control group). The initial RST scores of both groups fell within the same range, from 1.5 to 4.0 (mean $=2.22$ in the training group, mean $=2.19$ in the control group). Informed consent was obtained from each participant, in accordance with the protocol approved by the Advanced Telecommunications Research Institute International Brain Imaging Center Review Board. All participants were paid a modest honorarium for their participation in the study.

\section{VISUAL IMAGERY TRAINING PROCEDURE}

Specific training was provided in the form of a modified RST that required participants to read three sentences and remember three target words. During the training period, participants practiced imagining a target word by drawing a figure that represented the word. Drawing the figures required participants to switch their focus of attention from verbal to visual images. For example, as the sample protocol in Figure 1 shows, participants were asked to draw the figure of the jet plane using a black pencil when the target word indicated "travel."

In the first part of the training, as Figure 1 shows, participants read aloud one sentence and then drew a figure representing the target word on a piece of paper within $60 \mathrm{~s}$. Although producing these drawings was initially challenging, the task became easier with practice. In the second part of the training, participants were

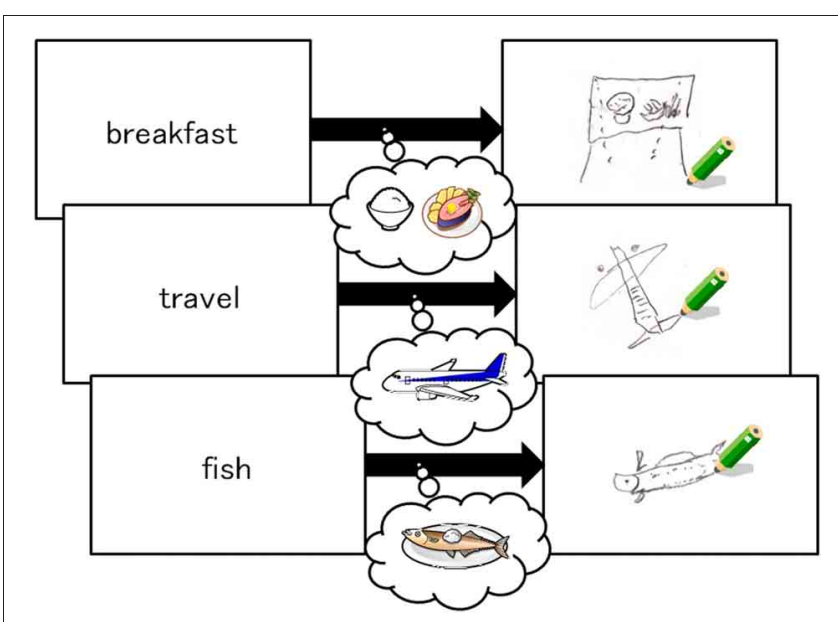

FIGURE 1 | Examples of participant's figure drawings (right) of the target word (left). 
asked to form a mental image of a figure representing the target word, instead of drawing the image on paper. Both parts of the practice training consisted of 20 trials composed of three sentences each and took approximately one hour. Participants in the control group read the same sentences and remembered the target words without being instructed to draw or imagine any figures. The number of trials was the same as the training group. Time was limited to $30 \mathrm{~min}$ in the first training session and $25 \mathrm{~min}$ in the second training session. The control group spent the same amount of time with the material as the training group. None of the participants in the training or control groups exceeded the time allotted.

\section{EXPERIMENTAL PROCEDURE}

Each participant was tested on the RST task during both preand post-training experimental sessions. The post-training RST experimental session was performed after the training (control training for the control group). The post-training session began approximately $2 \mathrm{~h}$ after the end of the pre-training session for both groups. In the experimental sessions, only three sentence trials were performed, and participants read each sentence silently. In the preliminary screening RST, most participants could not remember three target words while they read three sentences aloud. Therefore, we adapted a recognition method to make task performance easier. Participants were instructed to make recognition judgments about probe stimuli (i.e., deciding whether each stimulus was a target word or not) rather than recall the words orally. They were required to read three sentences silently while concurrently remembering three target words in each of the sentences. Each sentence appeared within $6 \mathrm{~s}$, and participants were required to push a button after they finished reading each sentence. After three sentences were presented for a given trial, three probe stimuli appeared at $6 \mathrm{~s}$ intervals in the same order as the sentences. Each probe stimulus comprised one word. When participants identified the target in the probe array, they pushed the left key to identify a word as "in set" and the right key to identify a word as "out of set." Half of the probe words were targets while the other half were not.

Figure 2 shows an RST experimental block (modified RST fitted to an fMRI experiment). One experimental RST block comprised three sentences in the reading phase (each lasting $18 \mathrm{~s}$ ) and an $18 \mathrm{~s}$ recognition phase. RST blocks were repeated 16 times in one session. A control condition lasting $18 \mathrm{~s}$ was inserted between experimental RST blocks. During the control condition, participants pushed the right and left keys when the words "right" or "left" appeared on the screen. Stimulus sentences ranged from 18 to 24 character lengths (mean $=21.5$ ) and were presented on the screen within a visual angle of $45^{\circ}$, using a mirror attached to a head coil. The target word that the participants were instructed to memorize was underlined in each sentence. The position of the target word in the sentence was counterbalanced across trials. RST blocks were repeated 16 times in post-training session, which was identical to the pre-training session. The RST and control conditions were repeated in the same order during the post-training session, but the stimulus sentences changed so that participants never read the same sentence twice.
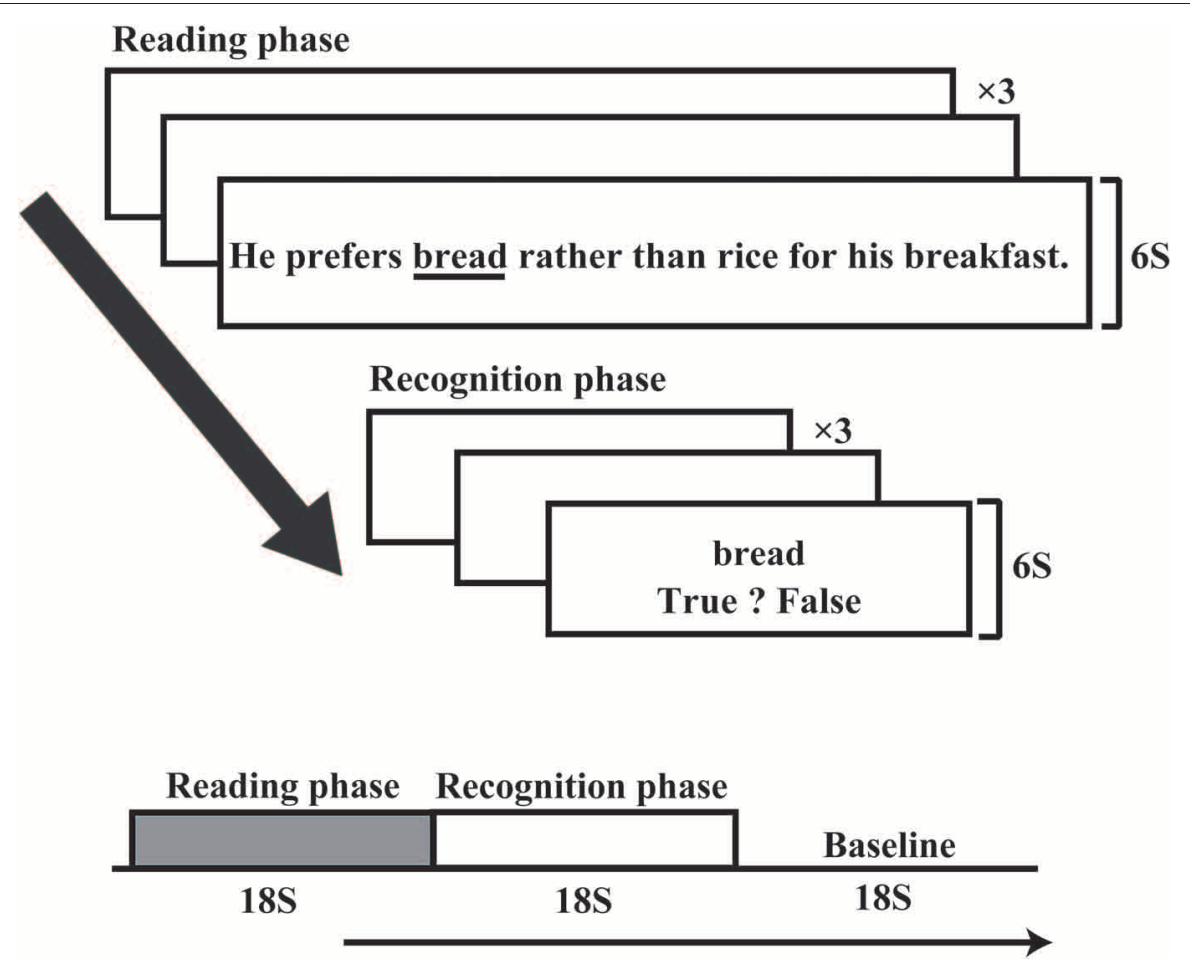

FIGURE 2 | Experimental time course of the RST. One experimental block comprised three sentences. After three sentences were presented, three probe stimuli appeared at $6 \mathrm{~s}$ intervals. Reading and recognition phases each took $18 \mathrm{~s}$, followed by an $18 \mathrm{~s}$ baseline condition. 


\section{fMRI DATA ACQUISITION AND ANALYSIS}

Whole brain imaging data were acquired on a 1.5-T MRI scanner (Shimazu-Marconi Magnex Eclipse), using a standard head coil. Head movements were minimized using a forehead strap. For functional imaging, a gradient-echo echo-planar imaging sequence with the following parameters was used: Repetition time (TR), $2000 \mathrm{~ms}$; echo time (TE), $48 \mathrm{~ms}$; flip angle, $80^{\circ}$; field of view (FOV), $22 \times 22 \mathrm{~cm}$; and matrix, $64 \times 64$ pixels. In one experimental session, 376 contiguous images and 20 slices each with a $6 \mathrm{~mm}$ thickness were obtained in the axial plane for each participant. After image collection, T1 anatomical images using a conventional spin echo pulse sequence $(\mathrm{TR}=12 \mathrm{~ms}, \mathrm{TE}=4.5 \mathrm{~ms}$, flip angle $=20^{\circ}, \mathrm{FOV}=25.6 \times 25.6 \mathrm{~cm}$, and pixel matrix $=$ $256 \times 256)$ were collected for anatomical co-registration at the same locations as the functional images. Scanner sequences were synchronized with stimulus presentation using Presentation stimulus software (Neurobehavioral System, Inc.). Data were analyzed using SPM2 (Wellcome Department of Cognitive Neurology, London, UK) on Matlab (MathWorks, Sherborn, MA).

Analysis of fMRI data was initially performed for each individual participant for each pre- and post-training session. Six initial images from each scanning session were discarded from analysis in order to eliminate non-equilibrium effects of magnetization, leaving 369 total images for analysis. All functional images were realigned to correct for head movement. We selected images with less than $1 \mathrm{~mm}$ movement between scans. After realignment, the anatomical images were co-registered to the mean functional images. Functional images were then normalized with the anatomical image and spatially smoothed using a Gaussian filter ( $7 \mathrm{~mm}$ full width-half maximum). The box-car reference function was adopted for individual analysis to identify voxels under each task condition. Global activity for each scan was corrected using grand mean scaling. Low-frequency noise was modeled using hemodynamic response functions and the corresponding derivative. Single participant data were analyzed using a fixedeffects model, whereas group data from pre- and post-training sessions were analyzed using a random-effects model. Extraction of ROI data and computation of percent signal change of each ROI was performed using MarsBaR (Brett et al., 2002). As a comparison between post- and pre-training sessions, we performed a paired $t$-test to examine the effect of training (post-training vs. pre-training) in each ROI. A statistical threshold of $p<0.05$ was used.

\section{RESULTS \\ BEHAVIORAL PERFORMANCE}

Recognition accuracy and response times during the experimental RST were calculated for both pre- and post-training phases. In the training group, the mean percentage recognition accuracies were higher in the post- than in the pre-training session (pre-, $90.33 \%, \mathrm{SD}=11.70$; post-, $97.60 \%, \mathrm{SD}=5.80)$. However, in the control group, there was little apparent difference between pretraining and post-training sessions (pre-, 90.43\%, SD = 10.70; post-, 91.20\%, SD = 9.13). A Two-Way analysis of variance (ANOVA) of group $\times$ session showed a significant main effect of training, $F(1,48)=6.26, p<0.05$. The interaction between group and session was also significant, $F(1,48)=9.59, p<0.01$, such that a significant increase in the post-training session was found only in the training group (Tukey's HSD post-hoc analysis, $p<0.01)$. Mean response times for the training group were $1454 \mathrm{~ms}(\mathrm{SD}=418.29)$ in the pre-training session and $1350 \mathrm{~ms}$ $(\mathrm{SD}=339.38)$ in the post-training session. The control group response times were $1543 \mathrm{~ms}(\mathrm{SD}=482.00)$ in the pre-training session and $1436 \mathrm{~ms}(\mathrm{SD}=409.00)$ in the post-training session. A Two-Way ANOVA showed a main effect of training, $F(1,48)=$ 19.60, $p<0.01$. There was a non-significant tendency toward an interaction between group and session, $F(1,48)=3.06, p<$ 0.08 . However, there was a significant post-training RT decrease in the training group (Tukey's HSD post-hoc analysis, $p<0.01$ ).

After the post-training session, all participants in the training group reported that they had used an imagery-based strategy to perform the post-training task. However, in the control group, most of the participants (20/25) used verbal rehearsal and five participants used both verbal rehearsal and visual imagery. These five participants did not show any performance increase.

\section{fMRI RESULTS}

Figure 3A shows areas of increased activation after training, compared using paired $t$-tests between the pre- and post-training sessions (voxel-level threshold uncorrected for multiple comparisons, $p<0.0001)$. We applied an uncorrected criterion to focus

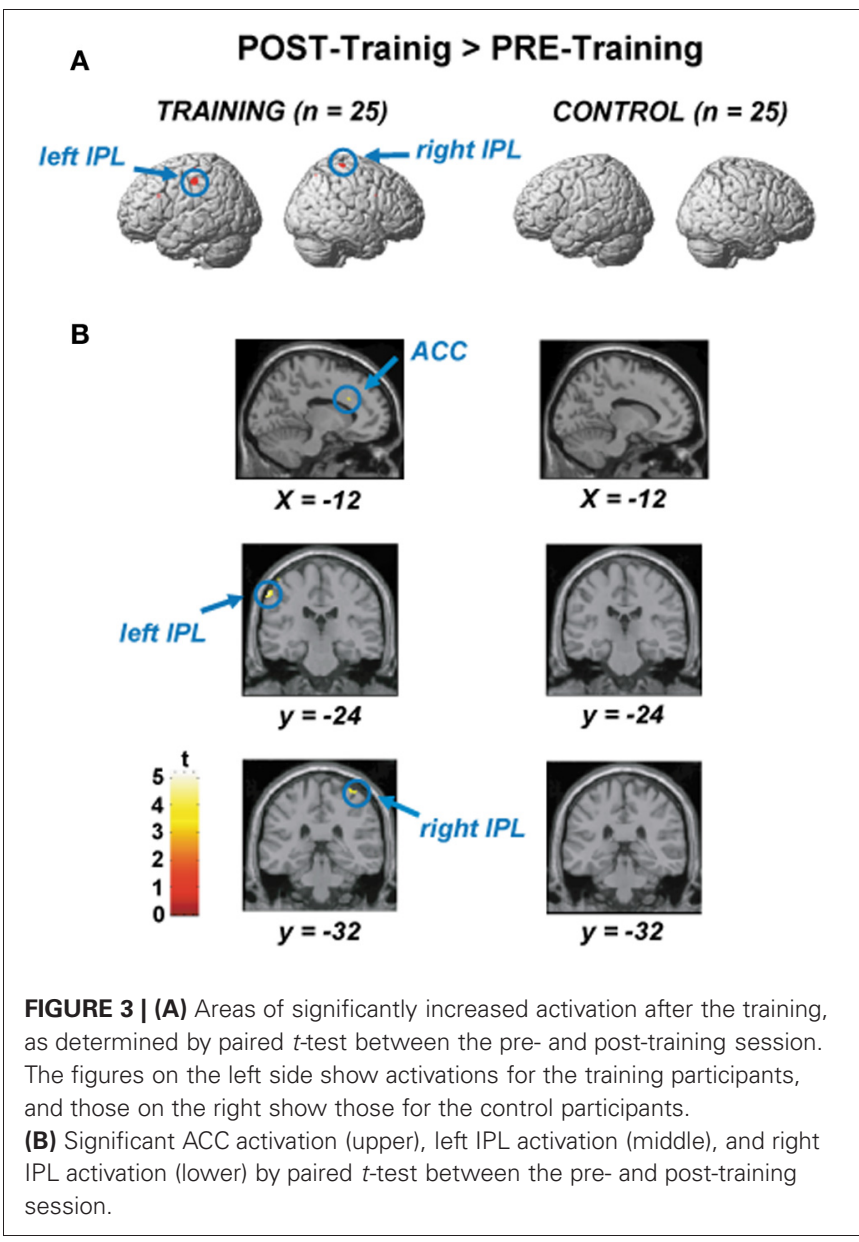


upon specific regions where increased activation had previously been reported for younger adults performing the RST (Bunge et al., 2000; Osaka et al., 2003, 2004). The figures on the left side show activations for training participants, and those on the right show activations for control participants.

Figure 3B displays areas for which there was significantly increased activation after training (voxel-level threshold uncorrected for multiple comparisons, $p<0.0001)$. Figure 3B shows significantly increased activation in the ACC $(x, y, z=$ $-12,22,28$, based on MNI coordinates, $Z$-score $=3.33$, $t$-value $=3.81, p<0.0001$, uncorrected), which was observed only in the training group. Increased activation during the posttraining session was also found in the left $(x, y, z=-60,-24,48$, $Z$-score $=4.22, t$-value $=5.22)$ and right inferior parietal lobule $(\mathrm{IPL})(x, y, z=40,-32,68, Z$-score $=3.49, t$-value $=4.03)$. An activation increase was also found in the right superior parietal lobule $(\mathrm{SPL})(x, y, z=30,-66,56, Z$-score $=3.14, t$-value $=$ 3.53; not shown in the Figure 3). In the control participants, we could not find any areas showing significant differences between pre- and post-training sessions. Furthermore, we could not find any areas for which there was significantly increased activation after training in the recognition phase both for experimental and control groups.

\section{SIGNAL INTENSITY}

The ACC, bilateral DLPFC, bilateral IPL, and bilateral SPL were specified as the regions of interest (ROI) for detecting signal changes. Following the identification of activated areas, percent signal changes in each ROI were obtained for both training and control groups. Time-course data were obtained for the local-maxima of each ROI for each participant. Mean percent signal change was calculated by subtracting the mean blood oxygenation-level-dependent (BOLD) signal at baseline from signals observed during the pre- and post-training sessions.

Figure 4 shows mean signal changes in each ROI during the pre- and post-training sessions in the training and control groups. Two-Way ANOVAs of group $\times$ session were performed for the ROIs. Analyses were performed using the STATISTICA software package (StatSoft). A significant interaction between session and group was found for the ACC region, $F(1,48)=6.93, p<0.01$. Training effects were found in the training group (Tukey's HSD post-hoc analysis, $p<0.05)$. A significant main effect of session $[F(1,48)=5.09, p<0.05]$ and an interaction between session and group $[F(1,48)=5.80, p<0.05]$ were also found for the left and right IPL. For the right IPL, training effects in signal intensity increases were found in the training group (by Tukey's HSD post-hoc analysis, $p<0.05$ ) but not the control group. Similarly, training effects were also found in the left IPL in the training group $(p<0.05)$ but not the control group. A significant main effect of session $[F(1,48)=7.04, p<0.05]$ and an interaction between session and group $[F(1,48)=4.80, p<$ 0.05] were also found for the right SPL, with the signal change actually increasing after training (Tukey's HSD post-hoc analysis, $p<0.05$ ). However, training effects in the recognition phase were not significant in these three regions for either the training group $[F(1,48)=1.13 \sim 2.23, p=0.26 \sim p=0.13]$ or the control group $[F(1,48)=0.81 \sim 2.24, p=0.37 \sim p=0.14]$.

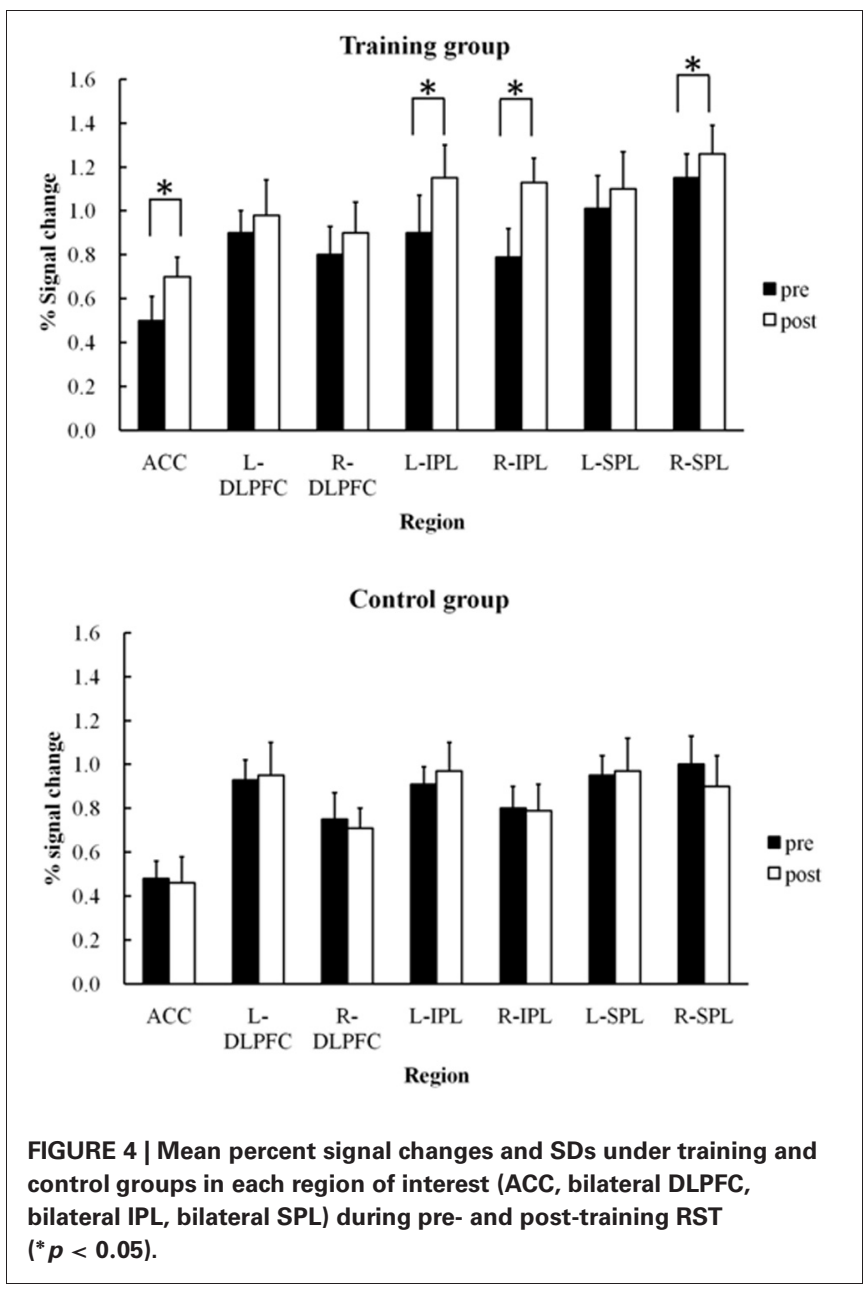

\section{DISCUSSION}

\section{INCREASING ACC RECRUITMENT}

In the present experiment, we found relatively scarce ACC activation during the pre-training RST, and these results were in accordance with previous findings in older adults (Otsuka et al., 2006). Interestingly enough, ACC activation significantly increased in the post-training RST, likely due to the effects of training. The ACC has an attention coordination function, including inhibiting attention directed toward inappropriate stimuli, whereas the DLPFC more generally supports sustaining attention on task goals (MacDonald et al., 2000). Increased ACC activity is also reported to occur with errors on highconflict trials such as go/no-go, oddball, and two-alternative forced-choice selections (Bush et al., 1998; Carter et al., 1998; Braver et al., 2001), suggesting that the ACC is active in conflict monitoring.

During the encoding phase of the RST in the scanner, participants needed to focus attention on the target words while inhibiting other irrelevant words in the sentence. Because the ACC potentially plays a role in perceiving conflicts between target and non-target words during the dual task, ACC impairment is associated with some deficits in exact executive control. Our participants likely did not have sufficient ACC activation in 
the pre-training session, leading to difficulties perceiving conflict between target and irrelevant words even if there was sufficient DLPFC activation to sustain attention to the target words. Therefore, without perceiving conflict during the encoding phase, participants face difficulty detecting differences between the target and non-target word during the recognition phase.

However, while participants performed the training task, they had to read one sentence at a time and then draw a figure representing the target word for each sentence. While they were drawing the figures (see Figure 1), participants could learn to switch attention from the target to its associated visual image code. Following such attention switching training (i.e., transferring from verbal to visual imagery), participant's likely experienced increased ACC activity as a function of increased attentional coordination. After the training, participants could more easily attend to targets and have more working memory resources available for both reading sentences and maintaining target words. With sufficient working memory resources, our participants could more easily assign the limited resources available for storage and processing during the RST.

Training appeared to promote ACC activation in the present study. With adequate coordination of attention, participants did not face a difficult conflict between the target and irrelevant filler words when they were required to recognize targets. Behavioral data also indicated better performance compared with performance during a pre-training RST. Moreover, response times decreased, which is seemingly indicative of increased confidence in correct responses after training.

\section{INCREASED ACTIVATION IN THE RIGHT IPL AND SPL}

The present PMRI data showed that right IPL and SPL activation was more apparent during the post-training RST compared to the pre-training RST. Retention of verbal information in the phonological loop itself activates the left IPL, while spatial information activates the right homologues (Smith et al., 1996). In post-training, all participants in the training group reported performing the task using verbal to visual code switching strategies. A similar result was reported by Carretti et al. (2007), who showed that strategic training using imagery to improve the memorization of word lists improved working memory performance in young and older individuals. According to Logie (2003), the visuo-spatial sketchpad can be subdivided into a visual cache, involved in storing visual images, and an inner scribe, involved in the retention of spatial information via active rehearsal (i.e., by drawing in the current study). The increased right IPL activation observed during the post-training RST may have been evoked by a visuo-spatial inner scribe that was used to sustain images of the target words (Logie and Pearson, 1997). Using the visuospatial inner scribe, participants can share the working memory load across two subsystems, providing them with a more efficient attentional coordination system.

It is interesting that providing a visually based imagery strategy improved performance on a verbal task in the present study. These findings suggest the intriguing possibility that when a verbal store within a multi-component model shows age-related performance declines, an alternative visual store can possibly serve to compensate for the deficits.
Right IPL and SPL activation is thought to be involved in the basic attentional process of executive function. A recent study demonstrated that right IPL activity is associated with disengagement and reorienting of attention to a relevant word presented outside the current focus of attention, whereas the intraparietal sulcus (IPS) contributes to an attentional set for the task goal (Corbetta et al., 2008). Furthermore, the right IPL (along with the SPL) likely plays a role in attentional filtering, which is a process whereby irrelevant information is screened out and relevant information prioritized (Minamoto et al., 2010).

Osaka et al. (2007) showed that increased left SPL activation coincided with attentional demands while performing a difficult RST requiring switching attention. While participants performed the difficult RST, the SPL appeared to play a role in reorienting attention and driving cooperative activity between the ACC and DLPFC. The SPL (including the lateral IPS area) is generally related to attentional processes (Culham and Kanwisher, 2001) and is specifically responsible for visuo-spatial attention and working memory (Wager and Smith, 2003). Activation in the posterior region supports the limited capacity of working memory (Linden, 2007). Thus, the SPL may potentially play a shared role in focusing attention along with DLPFC and ACC (Osaka and Osaka, 2007).

In the present study, bilateral activation was found in the IPL, which is often regarded as a mechanism that compensates for declining functional activity while performing working memory tasks (Cabeza et al., 2002; Reuter-Lorenz, 2002; Wager and Smith, 2003). It has also been suggested that reduced asymmetry in the PFC is compensatory when the homologous region can subserve a complementary role in task performance (Colcombe et al., 2005). When the attentional control system weakens, another system develops to compensate for any functional impairment. Bilateral IPL activation observed in the current study may serve as a compensatory system for impairments in working memory. In accord with previous suggestions (Wager and Smith, 2003; Colcombe et al., 2005), the observed right IPL activation possibly compensated for the declines in executive functioning with the help of the visuo-spatial inner scribe. This suggestion indicates that when one store becomes vulnerable to age-related decline, use of an alternative store, such as the visuo-spatial sketchpad, is a potentially reliable method to improve task performance in older adults. In the present experiment, we did not control the strategy use of the non-training group. If participants in the control group had adopted a visual imagery strategy, they might have shown a performance increase in behavioral and neural effects without training. Further investigation is needed to clarify the effects of strategy on working memory performance when only imagery instruction is provided. Moreover, the long-term effects of visual strategy training on working memory performance should be investigated in elderly populations. In addition, Grady et al. (2006) suggested that there is a gradual, age-related reduction in the ability to suspend non-task-related or defaultmode activity. Further study will be required to test default-mode activity in older individuals.

In sum, we found that training in a specific verbal to visual code switching strategy improved complex working memory task performance in older adults. After training, fMRI data showed 
an increase in ACC, bilateral IPL, and right SPL activation in the encoding phase.

Based on these findings, we suggest that age-related impairment in working memory performance is partially caused by ineffective attention coordination, as supported by the ACC in connection with the IPL and SPL. Training in dual task paradigms, which require compensatory strategies such as code switching, could be one potentially effective approach for coping with working memory deficits in aging.

\section{ACKNOWLEDGMENTS}

This work was supported by grants from the Japan Society for the Promotion of Science (\#23240036) and (\#22220003) to Mariko Osaka and Naoyuki Osaka, respectively.

\section{REFERENCES}

Baddeley, A. D. (1986). Working Memory. Oxford: Oxford University Press.

Baddeley, A. D. (2003). Working memory: looking back and looking forward. Nat. Rev. Neurosci. 4, 829-839.

Baddeley, A. D., and Logie, R. H. (1999). "Working memory: the multiple component model," in Models of Working Memory: Mechanisms of Active Maintenance and Executive Control, eds A. Miyake and P. Shah (Cambridge, MA: Cambridge University Press), 28-61.

Braver, T. S., Barch, D. M., Gray, J. R., Molfese, D. L., and Snyder, A. (2001). Anterior cingulate cortex and response conflict: effects of frequency, inhibition and errors. Cereb. Cortex 11, 825-836.

Brett, M., Anton, J. L., Valabregue, R., and Poline, J. B. (2002). "Region of interest analysis using an SPM toolbox," The $8^{\text {th }}$ International Conference on Functional Mapping of the Human Brain. June 2-6, Sendai, Japan. Available on CD-ROM in Neuroimage 16, Abstract 974.

Bunge, S. A., Klingberg, T., Jacobsen, R. B., and Gabrieli, J. D. (2000). A resource model of the neural basis of executive working memory. Proc. Natl. Acad. Sci. U.S.A. 97, 3573-3578.

Bush, G., Whalen, P. J., Rosen, B. R., Jenike, M. A., Mcinerney, S. C., and Rauch, S. L. (1998). The counting Stroop: an interference task specialized for functional neuroimagingvalidation study with functional MRI. Hum. Brain Mapp. 6, 270-282.

Cabeza, R. (2001). Cognitive neuroscience of aging: contributions of functional neuroimaging. Scand. J. Psychol. 42, 277-286.

Cabeza, R., Anderson, N. D., Locantore, J. K., and Mcintosh, A. R. (2002). Aging gracefully: compensatory brain activity in high-performing older adults. Neuroimage 17, 1394-1402.

Carretti, B., Borella, E., and De Beni, R. D. (2007). Does strategic memory training improve the working memory performance of young and older adults? Exp. Psychol. 54, 311-320.

Carter, C. S., Braver, T. S., Barch, D. M., Botvinick, M. M., Noll, D., and Cohen, J. D. (1998). Anterior cingulate cortex, error detection, and the online monitoring of performance. Science 280, 747-749.

Cohen, J. D., Perlstein, W. M., Braver, T. S., Nystrom, L. E., Noll, D. C., Jonides, J., and Smith, E. E. (1997). Temporal dynamics of brain activation during a working memory task. Nature 386, 604-608.

Colcombe, S. J., Kramer, A. F., Erickson, K. I., and Scalf, P. (2005). The implications of cortical recruitment and brain morphology for individual differences in inhibitory function in aging humans. Psychol. Aging 20, 363-375.

Corbetta, M., Patel, G., and Shulman, G. L. (2008). The reorienting system of the human brain: from environment to theory of mind. Neuron 58 , 306-324.

Craik, F. I. M., and Salthouse, T. A. (eds). (2000). The Handbook of Aging and Cognition. New Jersey: Lawrence Erlbaum.

Culham, J. C., and Kanwisher, N. G. (2001). Neuroimaging of cognitive functions in human parietal cortex. Curr. Opin. Neurobiol. 11, 157-163.

Dahlin, E., Neely, A. S., Larsson, A., Backman, L., and Nyberg, L. (2008a). Transfer of learning after updating training mediated by the striatum. Science 320, 1510-1512.

Dahlin, E., Nyberg, L., Backman, L. and Neely, A. S. (2008b). Plasticity of executive functioning in young and older adults: immediate training gains, transfer, and long-term maintenance. Psychol. Aging 23, 720-730.

Daneman, M., and Carpenter, P. A. (1980). Individual differences in working memory and reading. J. Verb. Learn. Verb. Behav. 19, 450-466.

D’Esposito, M., Detre, J. A., Alsop, D. C., Shin, R. K., Atlas, S., and Grossman, M. (1995). The neural basis of the central executive system of working memory. Nature 378, 279-281.
D'Esposito, M., Postle, B. R., Ballard, D., and Lease, J. (1999). Maintenance versus manipulation of information held in working memory: an event-related fMRI study. Brain Cogn. 41, 66-86.

Engle, R. W., Tuholski, S. W., Laughlin, J. E., and Conway, A. R. (1999). Working memory, short-term memory, and general fluid intelligence: a latent-variable approach J. Exp. Psychol. Gen. 128, 309-331.

Erickson, K. I., Colcombe, S. J., Wadhwa, R., Bherer, L., Peterson, M. S., Scalf, P. E., Kim, J. S., Alvarado, M., and Kramer, A. F. (2007). Training-induced plasticity in older adults: effects of training on hemispheric asymmetry. Neurobiol. Aging 28, 272-283.

Grady, C. L., Springer, M. V. Hongwanishkul, D., Mcintosh, A. R., and Winocur, G. (2006). Agerelated changes in brain activity across the adult lifespan. J. $\operatorname{Cog} n$. Neurosci. 18, 227-241.

Just, M. A., and Carpenter, P. A. (1992). A capacity theory of comprehension: individual differences in working memory. Psychol. Rev. 99, 122-149.

Li, S. C., Schmiedek, F., Huxhold O., Rocke, C., Smith, J., and Lindenberger, U. (2008). Working memory plasticity in old age: practice gain, transfer, and maintenance. Psychol. Aging 23, 731-742.

Linden, D. E. (2007). The working memory networks of the human brain. Neuroscientist 13, 257-267.

Logie, R. H. (2003). Spatial and visual working memory: a mental workspace. Psychol. Learn. Motiv. 42, 37-78.

Logie, R. H., and Pearson, D. G. (1997) The inner eye and the inner scribe of visuo-spatial working memory: evidence from developmental fractionation. Eur. J. Cogn. Psychol. 9, 241-257.

MacDonald, A. W. 3rd, Cohen, J. D., Stenger, V. A., and Carter, C. S (2000). Dissociating the role of the dorsolateral prefrontal and anterior cingulate cortex in cognitive control. Science 288, 1835-1838.

Madden, D. J., Whiting, W. L., Huettel, S. A., White, L. E., Macfall, J. R., and
Provenzale, J. M. (2004). Diffusion tensor imaging of adult age differences in cerebral white matter: relation to response time. Neuroimage 21, 1174-1181.

McNamara, D. S., and Scott, J. L. (2001). Working memory capacity and strategy use. Mem. Cognit. 29, $10-17$.

Minamoto, T., Osaka, M., and Osaka, N. (2010). Individual differences in working memory capacity and distractor processing: possible contribution of top-down inhibitory control. Brain Res. 1335, 63-73.

Nyberg, L., Sandblom, J., Jones, S., Neely, A. S., Petersson, K. M., Ingvar, M., and Backman, L. (2003). Neural correlates of training-related memory improvement in adulthood and aging. Proc. Natl. Acad. Sci. U.S.A. 100, 13728-13733.

Osaka, M., Komori, M., Morishita, M., and Osaka, N. (2007). Neural bases of focusing attention in working memory: an fMRI study based on group differences. Cogn. Affect. Behav. Neurosci. 7, 130-139.

Osaka, M., and Nishizaki, Y. (2000). "How working memory works in the central executive," in The Brain and Working Memory, ed N. Osaka (Kyoto: Kyoto University Press), 203-223.

Osaka, M., and Osaka, N. (2007). "Neural bases of focusing attention in working memory: an fMRI study based on individual differences," in The Cognitive Neuroscience of Working Memory, eds N. Osaka, R. H. Logie, and M. D'Esposito (Oxford: Oxford University Press), 99-118.

Osaka, M., Osaka, N., Kondo, H., Morishita, M., Fukuyama, H., Aso, T., and Shibasaki, H. (2003). The neural basis of individual differences in working memory capacity: an fMRI study. Neuroimage 18, 789-797.

Osaka, N., Osaka, M., Kondo, H., Morishita, M., Fukuyama, H., and Shibasaki, H. (2004). The neural basis of executive function in working memory: an fMRI study based on individual differences. Neuroimage 21, 623-631. 
Otsuka, Y., Osaka, N., Morishita, M., Kondo, H., and Osaka, M. (2006). Decreased activation of anterior cingulate cortex in the working memory of the elderly. Neuroreport 17, 1479-1482.

Otsuka, Y., Osaka, N., and Osaka, M. (2008). Functional asymmetry of superior parietal lobule for working memory in the elderly. Neuroreport 19, 1355-1359.

Owen, A. M., Stern, C. E., Look, R. B., Tracey, I., Rosen, B. R., and Petrides, M. (1998). Functional organization of spatial and nonspatial working memory processing within the human lateral frontal cortex. Proc. Natl. Acad. Sci. U.S.A. 95, 7721-7726.

Petersen, S. E., Fox, P. T., Posner, M. I., Mintun, M., and Raichle, M. E. (1988). Positron emission tomographic studies of the cortical anatomy of single- word processing. Nature 331, 585-589.

Posner, M. I., and Petersen, S. E. (1990). The attention system of the human brain. Annu. Rev. Neurosci. 13, 25-42.

Raz, N. (2000). "Aging of the brain and its impact on cognitive performance: integration of structural and functional findings," in The Handbook of Aging and Cognition, eds F. I. M. Craik and T. A. Salthouse (New Jersey: Lawrence Erlbaum), 1-90.

Raz, N., Gunning, F. M., Head, D., Dupuis, J. H., Mcquain, J., Briggs, S. D., Loken, W. J., Thornton, A. E., and Acker, J. D. (1997). Selective aging of the human cerebral cortex observed in vivo: differential vulnerability of the prefrontal gray matter. Cereb. Cortex 7, 268-282.

Reuter-Lorenz, P. (2002). New visions of the aging mind and brain. Trends Cogn. Sci. 6, 394.
Salthouse, T. A. (1996). The processingspeed theory of adult age differences in cognition. Psychol. Rev. 103 403-428.

Smith, E. E., and Jonides, J. (1999). Storage and executive processes in the frontal lobes. Science 283 1657-1661.

Smith, E. E., Jonides, J., and Koeppe, R. A. (1996). Dissociating verbal and spatial working memory using PET. Cereb. Cortex 6, 11-20.

Wager, T. D., and Smith, E. E. (2003). Neuroimaging studies of working memory: a meta-analysis. Cogn. Affect. Behav. Neurosci. 3, 255-274.

West, R. L. (1996). An application of prefrontal cortex function theory to cognitive aging. Psychol. Bull. 120, 272-292.

Yesavage, J. A. (1983). Imagery pretraining and memory training in the elderly. Gerontology 29, 271-275.
Conflict of Interest Statement: The authors declare that the research was conducted in the absence of any commercial or financial relationships that could be construed as a potential conflict of interest.

Received: 26 October 2011; accepted: 05 February 2012; published online: 20 February 2012

Citation: Osaka M, Otsuka Y and Osaka $N$ (2012) Verbal to visual code switching improves working memory in older adults: an fMRI study. Front. Hum. Neurosci. 6:24. doi: 10.3389/fnhum. 2012.00024

Copyright (c) 2012 Osaka, Otsuka and Osaka. This is an open-access article distributed under the terms of the Creative Commons Attribution Non Commercial License, which permits non-commercial use, distribution, and reproduction in other forums, provided the original authors and source are credited. 